\title{
BMJ Open Statins for the primary prevention of cardiovascular disease: an overview of systematic reviews
}

\author{
Paula Byrne, ${ }^{1}$ John Cullinan, ${ }^{1}$ Amelia Smith, ${ }^{2}$ Susan M Smith ${ }^{3}$
}

To cite: Byrne P, Cullinan J, Smith A, et al. Statins for the primary prevention of cardiovascular disease: an overview of systematic reviews. BMJ Open 2019;9:e023085. doi:10.1136/ bmjopen-2018-023085

- Prepublication history and additional material for this paper are available online. To view these files, please visit the journal online (http://dx.doi. org/10.1136/bmjopen-2018023085).

Received 22 March 2018 Revised 10 December 2018 Accepted 12 December 2018

Check for updates

(C) Author(s) (or their employer(s)) 2019. Re-use permitted under CC BY-NC. No commercial re-use. See rights and permissions. Published by BMJ.

${ }^{1}$ J.E. Cairnes School of Business and Economics, National University of Ireland Galway, Galway, Ireland

${ }^{2}$ Department of Pharmacology and Therapeutics, University of Dublin Trinity College, Dublin, Ireland

${ }^{3}$ HRB Centre for Primary Care Research and Department of General Practice, Royal College of Surgeons in Ireland, Dublin, Ireland

Correspondence to

Ms Paula Byrne;

pbyrne82@gmail.com

\section{ABSTRACT}

Objective To synthesise evidence from exclusively primary prevention data on the effectiveness of statins for prevention of cardiovascular disease (CVD), including stroke, and outcomes stratified by baseline risk and gender.

Design Overview of systematic reviews (SRs) using Revised-AMSTAR approach to assess quality.

Data sources Cochrane Database of Systematic Reviews, MEDLINE, Embase, PubMed, Scopus and PROSPERO to June 2017.

Eligibility criteria for selecting studies SRs of randomised control trials (RCTs) or individual patient data (IPD) from RCTs, examining the effectiveness of statins versus placebo or no treatment on all-cause mortality, coronary heart disease, CVD (including stroke) and composite endpoints, with stratification by baseline risk and gender.

Data extraction and synthesis Two independent reviewers extracted data and assessed methodological quality. A narrative synthesis was conducted.

Results Three SRs were included. Quality of included SRs was mixed, and none reported on the risk of bias of included trials. We found trends towards reduced allcause mortality in all SRs (RR 0.91 [95\% Cl 0.85 to 0.97]), (RR 0.91 [95\% Cl 0.83 to 1.01]) and (RR 0.78 [95\% Cl 0.53 to 1.15$]$ ) though it was not statistically significant in two SRs. When stratified by baseline risk, the effect on all-cause mortality was no longer statistically significant except in one medium risk category. One review reported significant reductions (RR 0.85 [95\% $\mathrm{Cl} 0.77$ to 0.95$]$ ) in vascular deaths and non-significant reductions in non-vascular deaths (RR 0.97 [95\% Cl 0.88 to 1.07]). There were significant reductions in composite outcomes overall, but mixed results were reported in these when stratified by baseline risk. These reviews included studies with participants considered risk equivalent to those with established CVD.

Conclusions There is limited evidence on the effectiveness of statins for primary prevention with mixed findings from studies including participants with widely ranging baseline risks. Decision making for the use of statins should consider individual baseline risk, absolute risk reduction and whether risk reduction justifies potential harms and taking a daily medicine for life.

Trial registration number CRD42017064761.

\section{INTRODUCTION}

Raised total cholesterol (TC) and low-density lipoprotein (LDL) cholesterol are risk

\section{Strengths and limitations of this study}

- Overview of systematic reviews that reviewed exclusively primary prevention data on statins.

- Transparent search strategy, published protocol and validated instruments to assess the methodological quality of included reviews.

- Synthesised evidence from systematic reviews with a mixture of individual patient and aggregate outcome data with an overlap of included randomised controlled trials across reviews.

- Some relevant reviews may have been excluded because we could not ascertain the proportion of primary prevention participants within the systematic review.

- Limited data reporting reductions in various outcomes based on baseline risk and some studies included participants considered risk equivalent to those with established cardiovascular disease.

factors for cardiovascular disease (CVD). ${ }^{1}$ Statins, or 3-hydroxy-3-methylglutaryl co-enzyme A reductase inhibitors, are a class of lipid-lowering drugs and are first choice agents for reducing plasma LDL cholesterol and thereby reducing CVD risk. ${ }^{2}$ Statins may be used for the primary or secondary prevention of CVD. Primary prevention comprises treating people who do not have established CVD but who may be at risk of future CVD events, whereas secondary prevention involves treating those with established CVD. ${ }^{3}$

The last 30 years have seen a large increase in the utilisation of statins, ${ }^{4-7}$ which is consistent with changes in recommendations in clinical guidelines. ${ }^{8}$ However, there is ongoing debate about these changes, ${ }^{9-11}$ as they have tended to expand the number of people eligible for treatment, particularly in primary prevention. ${ }^{12} 13$ Our previous analysis found that almost two-thirds of people who were taking statins did so for primary prevention. $^{14}$

Several SRs investigating the use of statins for the primary prevention of CVD have been published reaching varying conclusions. ${ }^{15-20}$ 
However, most published SRs reported on trials that included a proportion of participants with a history of CVD ${ }^{21-23}$ In addition, the primary prevention population is heterogeneous, ranging from those at very low risk of CVD to those considered 'risk equivalent' to those in the secondary prevention category. The latter, for example, includes people with diabetes mellitus exhibiting target organ damage or people with chronic kidney disease. ${ }^{1}$ The net benefit or absolute risk reduction achieved with statin therapy is critically dependent on the baseline risk. Therefore, the outcomes reported in the SRs that were stratified by baseline risk or by gender were of particular interest, these data being the most pertinent to clinical decision making with individual patients.

To address this evidence gap and support decision making, we undertook an overview of SRs that reported on exclusively primary prevention trials or individual patient data (IPD) of trial participants using only data from patients without established CVD.

\section{METHODS}

This overview was conducted according to the methods of the Cochrane Handbook for Systematic Reviews of Interventions. ${ }^{24}$ The protocol for the overview was published on PROSPERO. ${ }^{25}$

We included any SR of RCTs or IPD from RCTs, in any language, which examined the effectiveness of statins versus placebo or no treatment exclusively in those without prior CVD. We searched for the following outcomes: (1) all-cause mortality; (2) fatal and non-fatal coronary heart disease (CHD) and CVD events including stroke; (3) composite endpoints; and (4) any of these outcomes stratified by a calculation of future risk of CVD or by gender (see box 1).

The search strategy, terms and databases were chosen with the assistance of a health sciences librarian and are described in online supplementary appendix 1 , tables 1 and 2. We searched the Cochrane Database of Systematic Reviews, MEDLINE, Embase, PubMed, Scopus and PROSPERO from the date of the first statin's approval in $1987^{26}$ to June 2017.

Two overview authors (PB and AS) independently screened search results by title and abstract and obtained full-text versions of the articles identified by both as potentially relevant. $\mathrm{PB}$ and AS selected relevant articles by reading the full texts and applying the inclusion and exclusion criteria. PB and AS used Covidence systematic review software to manage the searches and extraction of data. ${ }^{27}$ Any differences of opinion on inclusion were resolved by consulting another overview author (SMS or JC).

\section{Data collection and analysis}

PB performed data extraction, while AS independently checked the extracted data. SRs were extracted that fulfilled inclusion criteria, that is, SRs of RCTs or IPD from RCTs, in any language, which examined the
Box 1 Patients, Interventions, Comparators, Outcomes, Timing, Setting and Study design

Patients
Adults $>18$ years of age.
Without established cardiovascular disease (CVD).
Interventions
Statins (3-hydroxy-3-methylglutaryl co-enzyme A reductase
inhibitors).
Comparators
Placebo.
Control.
Outcomes
All-cause mortality.
Fatal and non-fatal coronary heart disease (CHD), CVD and stroke
events.
Combined endpoint (fatal and non-fatal CHD, CHD and stroke
events).
Any of the above outcomes stratified by a calculation of future risk
of CVD or by gender.
Timing
Studies of any duration.
Setting and study design
Systematic reviews of randomised controlled trials.
Systematic reviews of individual patient data.

effectiveness of statins versus placebo or no treatment exclusively in those without prior CVD. We extracted data on outcomes of relevance; all-cause mortality; CHD, CVD and stroke events; composite endpoints; and any of these outcomes stratified by a calculation of future risk of CVD or by gender. Both reviewers independently assessed the methodological quality of the included reviews using the R-AMSTAR tool. ${ }^{28}$ One of the included SRs, published by the Cholesterol Treatment Trialists' Collaboration (CTT), consisted of analyses of IPD and was reported in two publications based on the same trials, one reporting results overall ${ }^{29}$ and one that included analyses stratified by gender. ${ }^{30}$ Some of the methods were described not in the SR itself but in other referenced CTT papers and in the CTT protocol, which was published in $1995 .{ }^{31-33}$ As we found some limitations in using R-AMSTAR in the context of IPD, we further assessed CTT and the secondary papers describing their methodology, using the Preferred Reporting Items for Systematic Reviews and Meta-analyses of Individual Participant Data (PRISMA-IPD) checklist. ${ }^{34}$ We searched the SRs for any assessments of the quality of evidence of included trials such as GRADE, Cochrane Risk of Bias or the Jadad Scale. We undertook a narrative synthesis of the included reviews and summarised their main results on the effectiveness of statins regarding the outcomes of relevance and those outcomes stratified by baseline risk and gender.

\section{Patient and public involvement}

There was no patient or public involvement in this overview, and patient and public involvement was not reported in the included systematic reviews. 


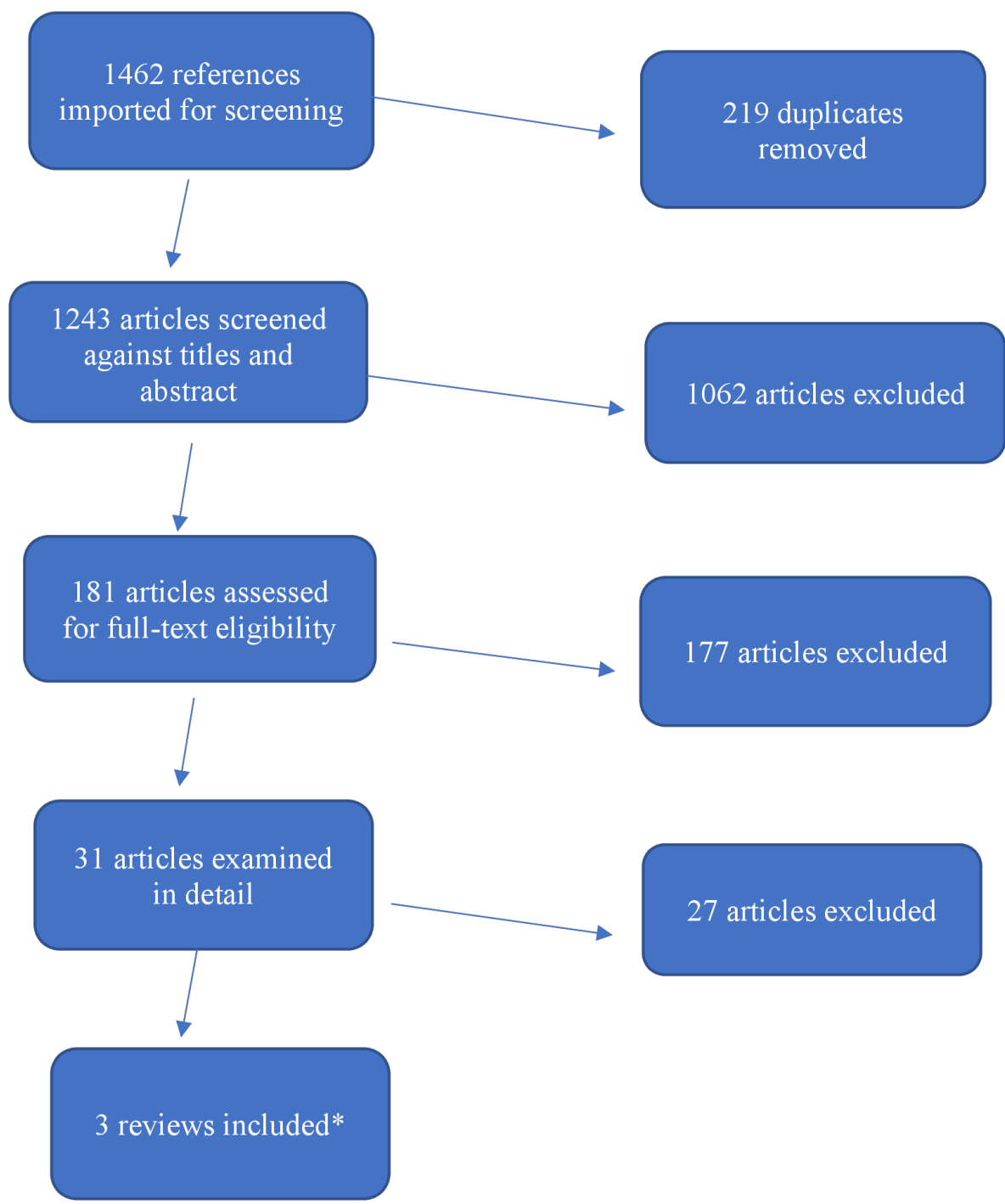

*One of the included systematic reviews comprised two publications from CTT analysis of IPD from the same trials

Figure 1 Flow chart of included systematic reviews. CTT, Cholesterol Treatment Trialists' Collaboration; IPD, individual patient data.

\section{RESULTS}

\section{Search results}

Our initial searches yielded 1462 results of which 181 were evaluated as full-text articles following title and abstract screening. Thirty-one full-text articles were analysed in detail. We supplemented the electronic search by scanning the reference lists of the full-text articles we read. No further SRs were identified. Reasons for exclusion and references to 27 final excluded articles are presented in online supplementary appendix 2. On the basis of our search and extraction strategy, three SRs were included in this overview. One of the included SRs was reported in two separate publications but included the same trial data, so was treated as a single systematic review for the purpose of this overview (see figure 1). Many excluded SRs did not specify the proportion of participants without CVD that had been included. Some primary prevention SRs included trials with up to $50 \%$ of participants with CVD, ${ }^{35}$ while the most up-to-date Cochrane review included trials with up to $10 \%$ of participants having CVD. ${ }^{21}$

\section{Characteristics of included studies}

Of the three included SRs, two comprised analyses of aggregate data from RCTs, ${ }^{15} 36$ and one ${ }^{29}{ }^{30}$ presented analyses of IPD from the CTT. Table 1 describes the characteristics of the included reviews. Table 2 describes the population demographics reported in each SR.

The CTT analyses included IPD from 22 trials of statin versus control as well as five of more versus less statin. However, the trials of more versus less statin did not include any primary prevention participants; therefore, 


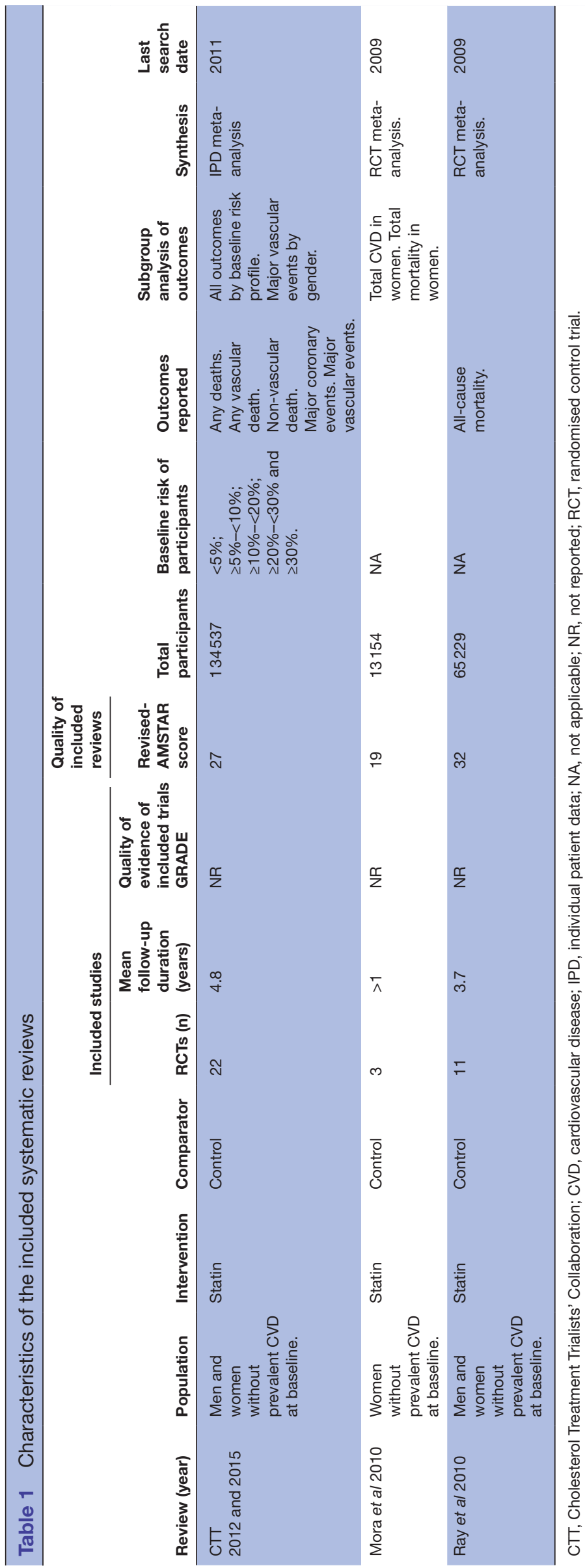

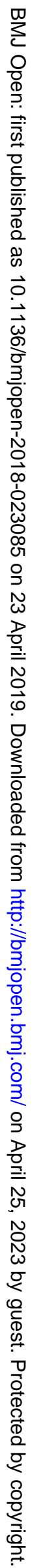




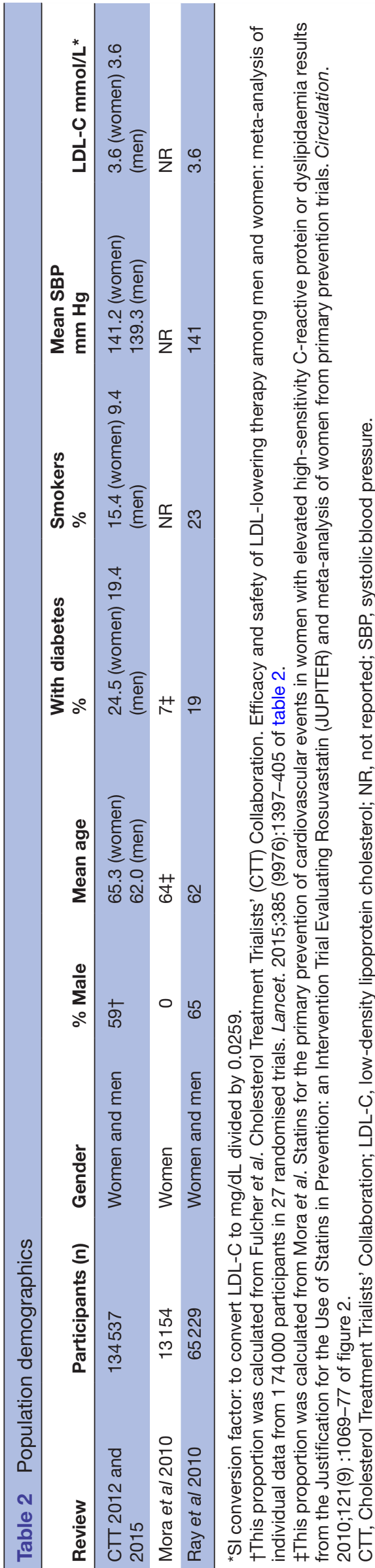

Table 3 Trial overlap of included systematic reviews

\begin{tabular}{|c|c|c|c|}
\hline Included trials & CTT & Mora & Ray \\
\hline AFCAPS/TexCAPS & $\checkmark$ & $\checkmark$ & $\boldsymbol{S}^{*}$ \\
\hline ALERT & $\checkmark$ & & \\
\hline ALLHAT-LLT & $\checkmark$ & & $\checkmark \dagger$ \\
\hline ALLIANCE & $\checkmark$ & & \\
\hline ASCOT-LLA & $\checkmark$ & & $\checkmark \dagger$ \\
\hline ASPEN & $\checkmark$ & & $\checkmark \dagger$ \\
\hline AURORA & $\checkmark$ & & \\
\hline CARDS & $\checkmark$ & & $\checkmark$ \\
\hline CARE & $\checkmark$ & & \\
\hline CORONA & $\checkmark$ & & \\
\hline $4 \mathrm{D}$ & $\checkmark$ & & \\
\hline GISSI-HF & $\checkmark$ & & \\
\hline GISSI-P & $\checkmark$ & & \\
\hline HPS & $\checkmark$ & & \\
\hline HYRIM & & & $\mathcal{S}^{*}$ \\
\hline JUPITER & $\checkmark$ & $\checkmark$ & $\checkmark$ \\
\hline LIPID & $\checkmark$ & & \\
\hline LIPS & $\checkmark$ & & \\
\hline MEGA & $\checkmark$ & $\checkmark$ & $\checkmark$ \\
\hline Post-CABG & $\checkmark$ & & \\
\hline PREVEND-IT & & & $\checkmark \dagger$ \\
\hline PROSPER & $\checkmark$ & & $\sqrt{ } \dagger$ \\
\hline $4 S$ & $\checkmark$ & & \\
\hline WOSCOPS & $\checkmark$ & & $\checkmark$ \\
\hline
\end{tabular}

*Provided hitherto unpublished tabular data on all-cause mortality. †Shared tabular data on subset of participants without CVD. CTT, Cholesterol Treatment Trialists' Collaboration; CVD, cardiovascular disease.

we assume, although it is not stated in the SR, that all analyses in those with 'no known history of vascular disease' are taken from only the statin versus control trials, and we included only these analyses in our overview as per our protocol. Online supplementary appendix 3 describes analyses from the included SRs that were not included in this overview.

Ray et $a l^{15}$ included 11 trials with additional unpublished data from authors that provided data on primary prevention participants. Mora $e t a \vec{l}^{36}$ included three exclusively primary prevention trials in women only. Two types of data therefore are included in our overview, IPD and aggregate data from trials.

The overlap of included reviews is reported in table 3 . While all SRs included overlapping RCTs, IPD were used by CTT, Ray et al reported previously unpublished data, and outcomes were reported differently across the three included systematic reviews. 


\section{Outcomes reported}

All three SRs reported outcomes for all-cause mortality, one of which, Mora et al, was in women only. CTT reported outcomes for vascular deaths, non-vascular deaths, major coronary events (defined as non-fatal myocardial infarction $[\mathrm{MI}]$ or coronary death) and major vascular events (defined as major coronary events, coronary revascularisation and stroke).

In addition, all outcomes reported by CTT were stratified by the participants' 5-year vascular risk at baseline. The risk categories reported in CTT describe a person's estimated 5-year risk of having a major vascular event and were stratified as follows: $<5 \%$; $\geq 5 \%-<10 \%$; $\geq 10 \%-<20 \%$; $\geq 20 \%-<30 \%$; and $\geq 30 \%$. This method of calculating risk was modelled by CTT, and how these categories relate to more commonly used methods such as SCORE, QRISK and Framingham is unclear. However, Robinson et a $\vec{p}^{77}$ estimated CTT's $\geq 5 \%-<10 \%$ 5-year vascular risk to be nearly identical to the more standard US 10-year atherosclerotic CVD (ASCVD) event rate. The outcome major vascular events was also stratified by gender by CTT, and Mora $e t$ al reported results for the composite outcome total CVD events in women. (See online supplementary appendix 3 for outcomes that were reported in the included SRs but which we did not include in this overview.)

\section{Quality assessment of the included systematic reviews R-AMSTAR}

The CTT SR received a R-AMSTAR score of 27. The reviews by Mora $e t$ al and Ray $e t$ al were assigned ratings of 19 and 32, respectively, out of a maximum score of 44 (see table 4).

In general, the included SRs scored lowest in criteria describing search strategies, excluded studies and reasons for exclusion. Neither Mora et alnor CTT clearly reported their search strategy, methods of study selection or extraction or provided lists and characteristics of excluded studies; nor how disagreements among extractors were resolved or if two independent researchers extracted data from the included studies. Across the included SRs, the highest scoring criterion was for the statistical methods of combining included studies.

Preferred Reporting Items for Systematic Reviews and Metaanalyses of Individual Participant Data

Using PRISMA-IPD as well as R-AMSTAR, we were still unable to assess potential risk of bias in the CTT SR including how IPD were collected, requested and managed, including whether IPD were sought and not available from other trials, the full electronic search strategy, details of databases searched, methods for resolving disagreements between those extracting studies, lists and characteristics of excluded studies, assessment of risk of bias in included RCTs and assessment of publication bias. It should be noted that this checklist was published in 2015, which is after the publication of CTT's SR. However, it would seem reasonable to expect that all items included in the PRISMA-IPD checklist would be

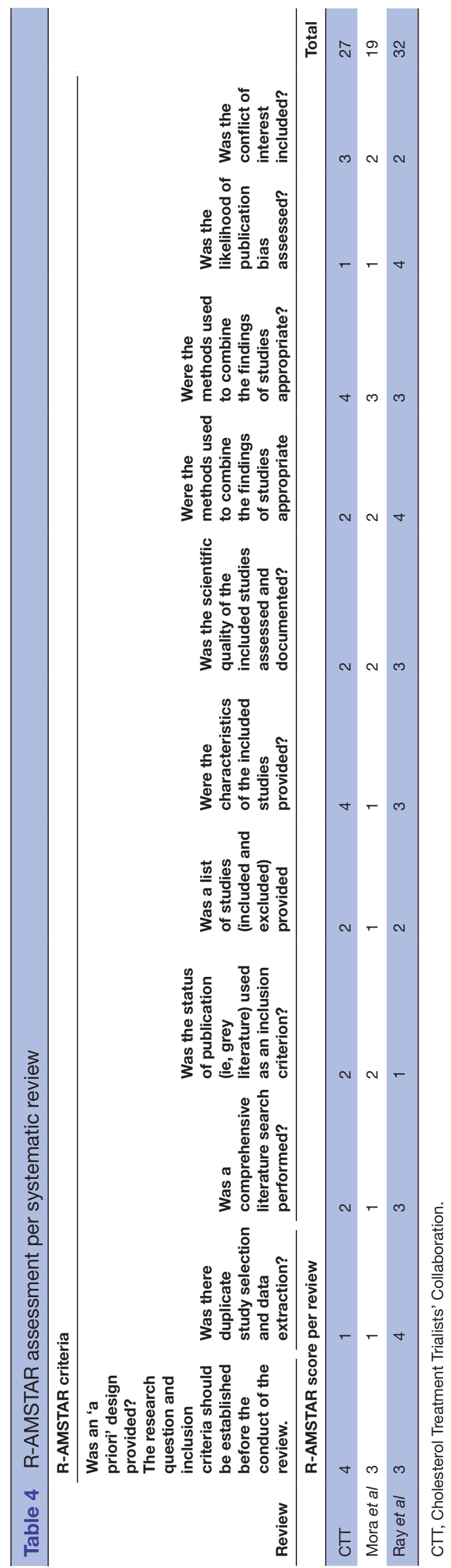


incorporated in IPD analysis. The CTT protocol notes that publication bias can be avoided by "prospectively planning an overview based on individual patient data from all relevant randomized trials'. However, no tests for publication bias were reported. Ray et al was the only included SR that reported on publication bias and assessed this using a funnel plot and Egger's test and found no strong evidence of publication bias $(\mathrm{p}=0.50)$.

\section{Risk of bias from the primary RCTs included in SRs}

None of the SRs reported on the risk of bias in their included primary trials using GRADE, Cochrane Risk of Bias or the Jadad Scale.

\section{Effectiveness of statins}

Due to the variability in the reviews and different outcomes reported, we have not attempted to combine or re-meta-analyse results and have presented a narrative synthesis as an overview of all results. We found a trend towards reduced all-cause mortality in the three systematic reviews, though only one of the three showed a statistically significant difference. CTT reported statistically significant relative risk (RR) reductions in 'any deaths' (RR 0.91 [95\% CI 0.85 to 0.97]). Ray et al conducted both fixed and random effects meta-analyses, with and without two trials reporting results for people with diabetes. There were no statistically significant reductions in all-cause mortality in any of the meta-analyses: random effects models (RR 0.91 [95\% CI 0.83 to 1.01]) including diabetes trials; fixed effects models (RR 0.93 [95\% CI 0.86 to 1.00]) including diabetes trials; random effects models (RR 0.92 [95\% CI 0.84 to 1.02]) excluding diabetes trials; fixed effects models (RR 0.94 [95\% CI 0.86 to 1.01]) excluding diabetes trials. Mora et al found no significant reduction in total mortality (RR 0.78 [95\% CI 0.53 to $1.15]$ ) in women.

CTT reported significant reductions in any vascular death (RR 0.85 [95\% CI 0.77 to 0.95 ]) and non-significant reductions in non-vascular death (RR 0.97 [95\% CI 0.88 to 1.07$]$ ).

In addition, CTT reported results stratified by baseline risk category. There were non-significant reductions reported by CTT in 'any deaths' except at one level of risk. Non-significant results for vascular deaths were reported in all risk categories. Three risk categories were found to have non-significant increases in non-vascular deaths, while two had non-significant reductions in the outcome. There were reductions reported in all risk categories for major coronary events and major vascular events when stratified by baseline risk category, but these were not statistically significant in the two highest risk categories (see table 5).

\section{DISCUSSION}

\section{Principal findings}

Three SRs were included in this overview reporting a mix of aggregate and IPD data and a range of reporting based on gender and baseline cardiovascular risk. The main outcomes reported were all-cause mortality, vascular and non-vascular deaths and the composite outcomes of total CVD events, major coronary events and major vascular events. CTT reported a significant reduction in all-cause mortality, but no significant reductions were found in the two other systematic reviews for this outcome. Though point estimates are very similar, the difference in statistical significance may be due to the numbers of participants included in each analysis, suggesting an issue with statistical power. It may be the case that the smaller analysis did not have sufficient statistical power though, conversely, it can be argued that when an analysis includes very large numbers, minimal clinical effects can reach statistical significance. ${ }^{38}{ }^{39}$ However, when CTT stratified results by baseline risk profile, non-significant reductions were reported for all-cause mortality in all but one level of risk. As noted, the overall reduction in any deaths reported in the reviews includes participants who, although categorised as 'primary prevention', may include those who are risk equivalent to people with established vascular disease, such as people with diabetes and chronic kidney disease. Because of this limitation, arguably the results relevant to low-risk people are those specific to their baseline risk category $(<5 \%$ and $\geq 5 \%-<10 \%)$, rather than the aggregate results reported.

In an attempt to specify risk reductions in the lowest risk people included in the CTT analysis, that is, in those for whom statins were not already recommended because of CHD risk equivalence, Abramson et $a l^{12}$ reanalysed data from this review for those whose 5-year risk was $<5 \%$ and for those whose risk was $\geq 5 \%-<10 \%$. They found there was no significant effect on mortality in this group of patients (RR 0.95 [95\% CI 0.86 to 1.04]). However, Abramson et als analysis included participants with and without vascular disease. It could be expected that the effect of statins would be seen most clearly in the outcome of vascular deaths. However, while an overall significant reduction was reported for this outcome by the CTT, non-significant reductions were reported at all levels of risk when stratified by baseline risk profile. Significant reduction in major vascular events and major coronary events were reported and mixed results for these outcomes when stratified by gender and baseline risk profile. It should be noted, however, that CTT's reporting of the composite outcome major vascular events is an addition to the outcomes prespecified in their protocol. No stroke outcomes were reported in any of the included SRs except as part of composite outcomes.

\section{Strengths and limitations of the overview}

As far as we are aware, this is the first overview of SRs that investigates statins in an exclusively primary prevention population. We synthesised evidence from SRs, which are considered the highest quality evidence for healthcare interventions. We used a transparent search strategy and followed a published protocol ${ }^{25}$ to guide our search, extraction and analysis. We used validated 


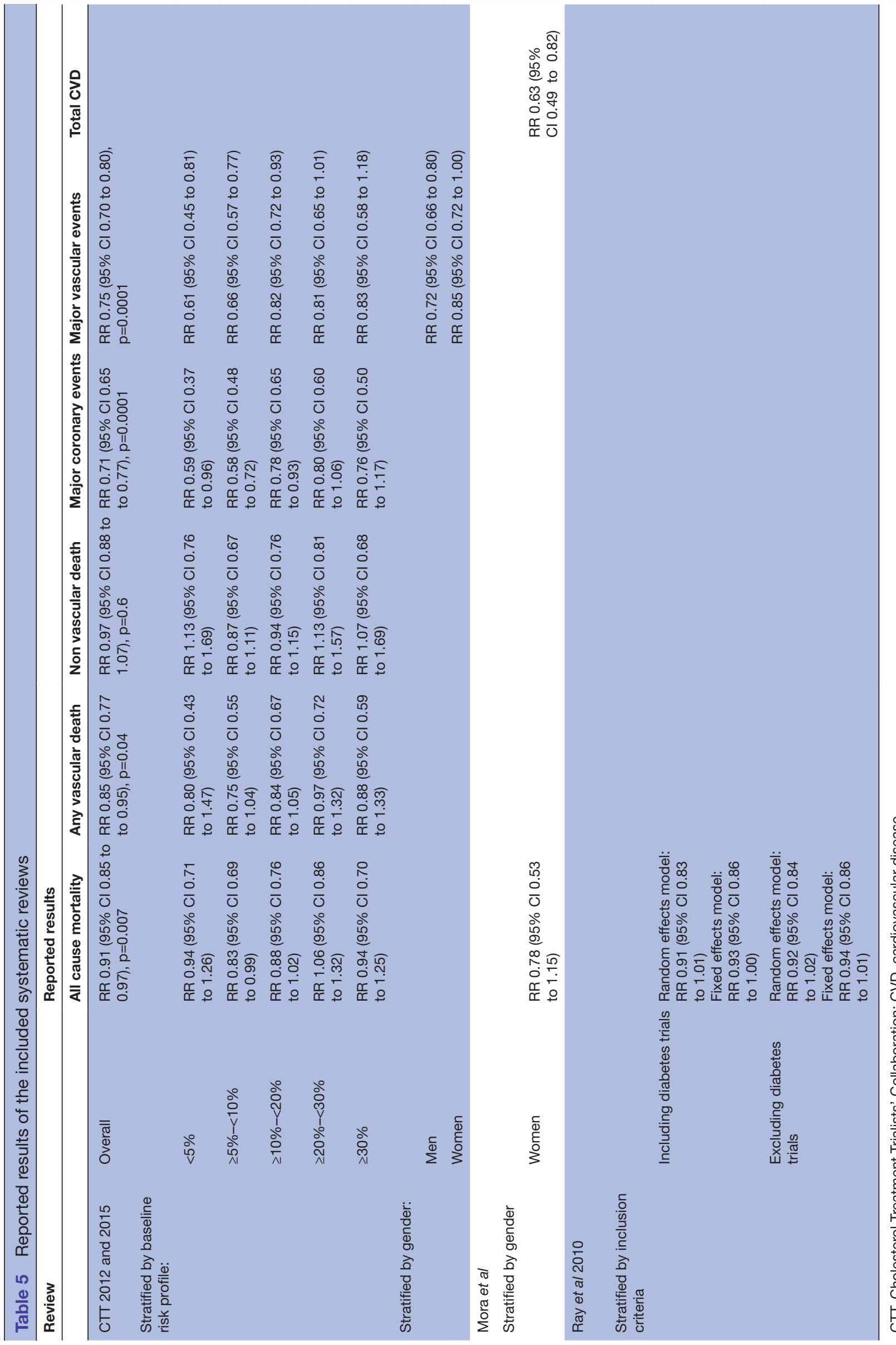

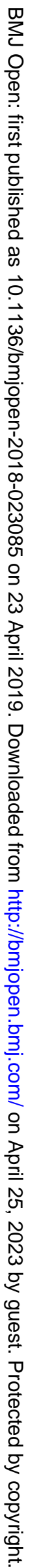


instruments to assess the methodological quality of included reviews. The main limitation of the overview is the need to synthesise evidence from SRs with a mixture of IPD and aggregate outcome data and the high level of overlap of included RCTs across reviews. Some trials are part of two or three of the included systematic reviews, whereas many others contribute only to the results of one systematic review. Therefore, the results of the analysis presented here may be driven mostly by these 'over-represented' populations. Some relevant reviews may have been excluded from this overview because we could not ascertain the proportion of primary prevention participants within the SR. Only one SR reported on risk of bias of the included trials, and we found some of the R-AMSTAR criteria ambiguous and difficult to answer. In addition, trials that fail to find significant benefit from an intervention are often not published, ${ }^{40}$ and it is possible that, as a result, our overview may be affected by publication bias. As we did not retrieve data from primary trials, we were limited to the information and judgements of the included SR authors. For example, the more recent primary prevention study, Heart Outcomes Prevention Evaluation-3 (HOPE-3), reported significant reductions in composite cardiovascular outcomes for those at 'intermediate risk' (defined as an annual risk of Major Vascular Events (MVEs) of approximately 1\%). ${ }^{41}$ Inclusion of this trial could have influenced the results reported in the included reviews in those in the intermediate risk category. Two of the included reviews had important methodological issues, notably the absence of clear reporting of search strategy and methods of study selection. This in fact is the core definition of a systematic review and warrants consideration of whether the individual included studies were simply reviews, as opposed to systematic reviews. In addition, the use of the composite outcomes by CTT was not prespecified in their 1995 protocol, and this change from the original protocol is not acknowledged or justified in the paper, which may introduce bias. ${ }^{42} 43$

Despite calls to make the provision of clinical trial data a legal, regulatory or ethical requirement, ${ }^{44} 45$ and specifically for the publication of CTT's IPD from statin trials, ${ }^{46} 47$ CTT's data, as well as much of the data from trials from the other two included reviews, remain unavailable for independent analysis, and thus, the goal of fully informed shared decision making cannot be achieved. In addition, while CTT analyses include data from 'almost all of the relevant randomised trials', ${ }^{48}$ members of the BMJ expert advisory group on statins stated that they intended to contact the authors of 183 statin trials for additional published and unpublished data. ${ }^{49}$ The inclusion of such data in systematic reviews and meta-analyses may alter reported results. However, though the gaps in the data cannot be overcome, such as the lack of transparency in the primary data, we believe this overview presents to patients and clinicians the best, although limited, data available.

\section{Clinical implications}

Some studies have shown that, in absolute terms, the majority of statins users are in the primary prevention category. ${ }^{50}$ A higher proportion of women who take statins fall into the primary prevention category than men, ${ }^{50}{ }^{51}$ and the distribution of statin prescribing has shifted from secondary to primary prevention particularly among women. ${ }^{52-54}$ Given the ongoing debate on the appropriateness of statin use in primary prevention, ${ }^{51} 55$ it is surprising that so few systematic reviews of exclusively primary prevention data exist. Clinical guidelines do not inform the physician whether recommended thresholds represent valid demarcation lines in terms of the individual patient, ${ }^{56}$ and decisions to take or prescribe a medicine involve a trade-off between the perceived benefits and harms of that medicine for the individual. This trade-off is particularly salient for low-risk people choosing to take a statin for primary prevention of CVD as the patient often feels healthy and may perceive the medicine as unnecessary, with uncertain benefits and potential side effects. Conversely, it may be the case that clinicians and patients would desire a reduction in CVD, regardless of how small, if they can tolerate statins. Therefore, for people at low-risk of CVD, it is important that the decision to prescribe or take statins is considered in terms of absolute risk reduction to ensure the potential benefits outweigh the potential harms in the context of that patient's preferences. Unfortunately, some of the gaps in the data we have presented here cannot be overcome. Only one included review stratified patients by risk and gender and one by gender only. The question remains for the clinician: what is the relevant information for the individual patient?

Arguably, clinical decisions should be based on 'hard' endpoints such as cardiovascular death, MI and stroke because these are least subject to bias in adjudication. ${ }^{12}$ As these outcomes were not reported separately in the overview, 'all-cause mortality' is the most reliable outcome on which to base decisions. The use and reporting of composite outcomes has been criticised as they may be unreasonably combined, inconsistently defined and inadequately reported. ${ }^{57}$ Reported risk reductions of composite outcomes may be driven by large reduction in the less serious components of the outcome rather than the more serious. For example, if a composite outcome comprises a larger proportion of 'less serious' outcomes such as angina and revascularisations compared with MIs or stroke, this may result in misleading impressions of the impact of treatment. ${ }^{36}$ Mora et al analysed the components of the composite outcome total CVD events in women in one large trial included in their review and found that women had a significant reduction in revascularisations and unstable angina but not in other components of the composite outcome, including stroke. Patients or prescribers may alter their decision making about the potential benefits of statin use even though larger treatment effects may be associated with less important components. ${ }^{58}$ Data for each component of each composite 
outcome in the included reviews were not supplied and some of the meta-analyses included composite outcomes, which may be inappropriate. ${ }^{57}$ The details of composite outcomes in the included SRs are described in online supplementary appendix 4 .

For the individual patient and clinician, there are three considerations in the process of informed decision making. First, what is the RR reduction according to the baseline risk of the individual. Second, what is the absolute risk reduction in risk for that person and, finally, what are potential side effects from taking statins in the context of that patient's preferences.

As we have outlined in this overview, the outcomes reported in the SRs that were stratified by baseline risk or by gender may be the most pertinent to clinical decision making. For example, for a woman whose risk is $<5 \%$, which results are relevant? Should she be presented with the relevant non-gender-specific results reported by CTT, such as the overall results for relative reduction in all-cause mortality (RR 0.91 [95\% CI 0.85 to 0.97 ]) or the non-significant relative reductions for those at her relevant baseline risk (RR 0.94 [95\% CI 0.71 to 1.26 ])? Or should she be presented with the overall non-significant relative reduction presented by Mora et al for women (RR 0.78 [95\% CI 0.53 to 1.15 ])? The same dilemma would arise for a high-risk woman, for example, one whose baseline risk is $30 \%$ or greater. In this case, the relevant risk reduction reported at her baseline risk was RR 0.94 (95\% CI 0.70 to 1.25 ). In a discussion on how to apply results of systematic reviews to patient care, Murad et $a t^{40}$ suggest that clinicians consider the upper and lower bounds of CIs. They can then consider how they would advise their patients were the upper boundary to represent the truth and how they would advise their patients were the lower boundary to represent the truth.

The included SRs reported reductions in risk of CVD outcomes as RR reductions, but for an individual patient, knowing their absolute risk reduction is more relevant when making a decision to take a statin. ${ }^{40}$ Sun $e t a \bar{l}^{59}$ give a good example of two people for comparison. One is a 65-year-old man who smokes, does not have heart disease but who has high total cholesterol levels and elevated blood pressure. The second is a 45-year-old woman who does not smoke, has elevated total cholesterol levels and slightly elevated blood pressure. Based on the American College of Cardiology/American Heart Association (ACC/AHA) risk calculator, the man has a $38 \%$ absolute risk of having a major coronary event in the next 10 years; the woman has a $1.4 \%$ absolute risk. According to the risk reductions reported by CTT in this overview, ${ }^{29}$ statin therapy would reduce the man's RR of major coronary events by $24 \%$ and the woman's RR by $41 \%$. However, the man could expect an absolute risk reduction of about $9 \%$ (number needed to treat of 11) and the woman of $0.6 \%$ (number needed to treat of 166) (online supplementary appendix 5).

Having considered which RR reduction is most relevant to the particular individual and the associated absolute risk reduction, the next consideration should be the potential harms from taking statins. Collins et al have reported that treating 10000 patients for 5 years would cause about five cases of myopathy, 50-100 new cases of diabetes and 5-10 haemorrhagic strokes. The authors argue that the harmful effects of statin therapy can usually be reversed without residual effect by stopping the statin therapy, whereas 'harmful effects of heart attacks or strokes that occur because statin therapy has not been used can be devastating, ${ }^{60}$ Even if there are side effects in a lower risk person, they may derive long-term benefit by stabilising or slowing the progression of subclinical vascular disease. However, the scale of these benefits may not justify the potentially wider effect of medicalisation of low-risk individuals. In addition, this definition of myopathy, as described by Armitage et al, ${ }^{61}$ may be high bar for diagnosing muscle symptoms among real people who may simply define myopathy as any muscle symptom, and observational data suggest that the frequency of statin myopathy may be higher. ${ }^{62}{ }^{63}$ Indeed, there is a large difference between the quantification of muscle side effects between Collins et al and Buettner et al, the former reporting five cases of myopathy per 10000 treated patients over 5 years, while the latter, a difference of $5.3 \%$ between statin and placebo groups, which is the equivalent of 530 cases of 'musculoskeletal pain' per 10000 patients treated. Thus, the estimates vary by a factor of 100. However, a recent systematic review of observational studies on statins use and new-onset diabetes noted that this association may be limited due to 'indication bias ${ }^{\text {,64; }}$; that is, the risk of an adverse event is related to the indication for medication use but not the use of the medication itself. In an observational study, one can only observe the effect of an exposure, in this case to statins. Groups are not randomly assigned to treatment or placebo groups. Therefore, observational studies are much more vulnerable to confounding bias, and their results may be less robust than those of a randomised controlled trial. ${ }^{65}$ For example, prediabetes, the most important risk factor for type 2 diabetes, is associated with dyslipidaemia, and this increases both the chances that people with prediabetes will be treated with statins and that these subjects will develop type 2 diabetes. ${ }^{66}$ Moreover, those with type 2 diabetes are considered risk equivalent to those in the secondary prevention category and their treatment with statins recommended by clinical guidelines. ${ }^{1}$ Consideration of the potential risk of developing type 2 diabetes from statin use is complex. The small risk of developing diabetes may be favourably balanced by the cardiovascular benefit. ${ }^{67}$ In addition, although those with diabetes have a higher cardiovascular event rate than those without, it may be the case that the event rate in those with new-onset diabetes is lower than those with established diabetes at baseline. ${ }^{68}$ This would strengthen the argument that any potential risk of new-onset diabetes is outweighed by the lowering of cardiovascular risk.

A recent systematic review by Albarqouni et $a t^{69}$ attempted to quantify the minimum acceptable risk 
reduction that patients say is necessary to justify a daily intake of medication to prevent CVD events. Our forthcoming analysis (Byrne et al) ${ }^{70}$ of those considered low, medium, high and very high risk according to the most recent 2016 guideline found that only some of those at high or very high risk would reach an acceptable level of risk reduction to justify taking a medicine for life. In addition, Albarqouni et al reported that in one study only $3 \%$ of community living older people would agree to CVD preventative medicines if that medication had adverse effects that could affect their activities of daily living and half would not agree to take the medication if it was associated with even mild fatigue or nausea. ${ }^{70}$

\section{Comparison with other studies}

We are aware of only one other overview of systematic reviews on the topic of statins for the primary prevention of CVD by Karmali et al. ${ }^{71}$ This overview considered drugs, including statins, for the primary prevention of CVD and reported statistically significant reductions in the risk of CVD for statins (RR 0.75 [95\% CI 0.70 to 0.81 ]). The Karmali et al overview included three of the four reviews from our overview. ${ }^{1529} 30$ However, no attempt was made to disaggregate exclusively primary prevention data, and therefore, it cannot be compared with our overview.

\section{CONCLUSION}

This overview suggests there is mixed evidence on the effectiveness of stains in primary prevention populations; however, this population is heterogeneous with widely ranging baseline risks. For the individual patient and clinician, there are three considerations in the process of informed decision making. First, what is the RR reduction according to the baseline risk of the individual. Second, what is the absolute risk reduction in risk for that person, and finally, what are potential side effects from taking statins in the context of that patient's preferences. The gaps in the data, outlined in this overview, cannot be overcome without patient-level data being made available for independent analysis. However, this overview clearly elucidates the information that is available at present allowing patients and clinicians to know that this is the best, although limited, data available.

Acknowledgements We would like to thank health sciences librarian Ms Jane Mulligan and Ms Edel Murphy of the HRB Clinical Research Facility and the Primary Care Clinical Trials Network Ireland in NUI Galway. Thanks also to biostatistician Dr Finbarr Lacey of RCSI, Dublin, for his help with the quality assessment tools.

Contributors PB was the lead researcher and involved in the design, implementation and analysis and reporting of the overview. PB conducted the initial search for systematic reviews. PB and AS undertook title and abstract screening, full-text screening, extraction of data and quality assessment. SMS arbitrated any disagreements between PB and AS on inclusion and quality assessment of reviews. $\mathrm{JC}$ and SMS provided substantial contributions to the conception, design, analysis and reporting of the work. All authors have read and approved the final manuscript and agree to be accountable for all aspects of the work. PB is the guarantor of this paper.

Funding This overview is part of PB's PhD, which is funded by the SPHeRE HRB structured PhD programme (HRB SPHeRE/2013/1). AS is funded by the Irish Cancer Society Collaborative Cancer Research Centre BREAST-PREDICT (CCRC13GAL). All authors had full access to the data and had final responsibility for the decision to submit this publication.

Competing interests None declared.

Patient consent for publication Not required.

Provenance and peer review Not commissioned; externally peer reviewed. Data sharing statement № additional data are available.

Open access This is an open access article distributed in accordance with the Creative Commons Attribution Non Commercial (CC BY-NC 4.0) license, which permits others to distribute, remix, adapt, build upon this work non-commercially, and license their derivative works on different terms, provided the original work is properly cited, appropriate credit is given, any changes made indicated, and the use is non-commercial. See: http://creativecommons.org/licenses/by-nc/4.0/.

\section{REFERENCES}

1. Catapano AL, Graham I, De Backer G, et al. 2016 ESC/EAS Guidelines for the Management of Dyslipidaemias. Eur Heart $J$ 2016;37:2999-3058.

2. Taylor F, Huffman MD, Macedo AF, et al. Statins for the primary prevention of cardiovascular disease. Cochrane Database Syst Rev 2013;1.

3. Perk J, De Backer G, Gohlke H, et al. European Association for Cardiovascular Prevention \& Rehabilitation (EACPR); ESC Committee for Practice Guidelines (CPG). European Guidelines on cardiovascular disease prevention in clinical practice (version 2012). The Fifth Joint Task Force of the European Society of Cardiology and Other Societies on Cardiovascular Disease Prevention in Clinical Practice (constituted by representatives of nine societies and by invited experts). Eur Heart J 2012;33:1635-701.

4. Walley T, Folino-Gallo P, Stephens P, et al. Trends in prescribing and utilization of statins and other lipid lowering drugs across Europe 1997-2003. Br J Clin Pharmacol 2005;60:543-51.

5. Feely J, McGettigan P, Kelly A. Growth in use of statins after trials is not targeted to most appropriate patients. Clin Pharmacol Ther 2000;67:438-41.

6. Kildemoes HW, Støvring H, Andersen M. Driving forces behind increasing cardiovascular drug utilization: a dynamic pharmacoepidemiological model. Br J Clin Pharmacol 2008;66:885-95.

7. DeWilde S, Carey IM, Bremner SA, et al. Evolution of statin prescribing 1994-2001: a case of agism but not of sexism? Heart 2003;89:417-21.

8. Moynihan RN, Cooke GP, Doust JA, et al. Expanding disease definitions in guidelines and expert panel ties to industry: a crosssectional study of common conditions in the United States. PLOS Med 2013;10:e1001500.

9. Greenland P, Bonow RO. Interpretation and use of another statin guideline. JAMA 2016;316:1977-9.

10. Redberg RF, Katz MH. Statins for primary prevention: the debate is intense, but the data are weak. JAMA 2016;316:1979-81.

11. Unruh L, Rice T, Rosenau PV, et al. The 2013 cholesterol guideline controversy: Would better evidence prevent pharmaceuticalization? Health Policy 2016;120.

12. Abramson JD, Rosenberg HG, Jewell N, et al. Should people at low risk of cardiovascular disease take a statin? BMJ 2013;347:f6123.

13. Goldacre B, Smeeth L. Mass treatment with statins. Br Med J 2014;349:g4745.

14. Byrne P, Cullinan P, Murphy $C$, et al. Cross-sectional analysis of the prevalence and predictors of statin utilisation in Ireland with a focus on primary prevention of cardiovascular disease. BMJ Open 2018;8:e018524.

15. Ray KK, Seshasai SR, Erqou S, et al. Statins and all-cause mortality in high-risk primary prevention: a meta-analysis of 11 randomized controlled trials involving 65,229 participants. Arch Intern Med 2010;170:1024-31.

16. de Lorgeril M, Rabaeus M. Beyond confusion and controversy can we evaluate the real efficacy and safety of cholesterol-lowering with statins? JCBMR 2016;1:67-92.

17. Ijioma N, Robinson JG. Statins and primary prevention of cardiovascular disease in women a critical appraisal of the evidence. Am J Lifestyle Med 2015;9:114-29.

18. Petretta M, Costanzo P, Perrone-Filardi $\mathrm{P}$, et al. Impact of gender in primary prevention of coronary heart disease with statin therapy: a meta-analysis. Int J Cardiol 2010;138:25-31.

19. Brugts JJ, Yetgin T, Hoeks SE, et al. The benefits of statins in people without established cardiovascular disease but with cardiovascular 
risk factors: meta-analysis of randomised controlled trials. BMJ 2009;338:b2376.

20. de Vries FM, Denig P, Pouwels KB, et al. Primary prevention of major cardiovascular and cerebrovascular events with statins in diabetic patients: a meta-analysis. Drugs 2012;72:2365-73.

21. Taylor FC, Huffman M, Ebrahim S. Statin therapy for primary prevention of cardiovascular disease. JAMA 2013;310:2451-2.

22. Chou R, Dana T, Blazina I, et al. Statins for prevention of cardiovascular disease in adults: evidence report and systematic review for the US Preventive services task force. JAMA 2016;316:2008-24

23. Thavendiranathan $P$, Bagai $A$, Brookhart MA, et al. Primary prevention of cardiovascular diseases with statin therapy: a meta-analysis of randomized controlled trials. Arch Intern Med 2006;166:2307-13.

24. Higgins JP, Green S. Cochrane handbook for systematic reviews of interventions: John Wiley \& Sons, 2011.

25. Byrne P, Cullinan J, Smith A, et al. Statins for the primary prevention of cardiovascular disease: an overview of systematic reviews. 2017.

26. Endo A. The Origin of the Statins. International Congress Series: Elsevier, 2004.

27. Veritas Health Innovation. www.covidence.org.

28. Kung J, Chiappelli F, Cajulis OO, et al. From systematic reviews to clinical recommendations for evidence-based health care: validation of revised assessment of multiple systematic reviews (r-amstar) for grading of clinical relevance. Open Dent $J$ 2010;4:84-91.

29. Mihaylova B, Emberson J, Blackwell L, et al. The effects of lowering LDL cholesterol with statin therapy in people at low risk of vascular disease: meta-analysis of individual data from 27 randomised trials. Lancet 2012;380:581-90.

30. Fulcher J, O'Connell R, Voysey M, et al. Cholesterol Treatment Trialists'(CTT) Collaboration. Efficacy and safety of LDL-lowering therapy among men and women: meta-analysis of individual data from 174,000 participants in 27 randomised trials. Lancet 2015;385:1397-405.

31. Baigent C, Keech A, Pa K, et al. Cholesterol Treatment Trialists'(CTT) Collaborators. Efficacy and safety of cholesterol-lowering treatment: prospective meta-analysis of data from 90,056 participants in 14 randomised trials of statins. Lancet 2005;366:1267-78.

32. Baigent $\mathrm{C}$, Blackwell L, Emberson J, et al. Efficacy and safety of more intensive lowering of LDL cholesterol: a meta-analysis of data from 170,000 participants in 26 randomised trials. Lancet 2010;376:1670-81.

33. Downs J, Gotto A, Clearfield M, et al. Protocol for a prospective collaborative overview of all current and planned randomized trials of cholesterol treatment regimens. Am J Cardiol 1995;75:1130-4.

34. Stewart LA, Clarke M, Rovers $M$, et al. Preferred reporting items for systematic review and meta-analyses of individual participant data: the PRISMA-IPD statement. JAMA 2015;313:1657-65.

35. Mills EJ, Rachlis B, Wu P, et al. Primary prevention of cardiovascular mortality and events with statin treatments: a network metaanalysis involving more than 65,000 patients. J Am Coll Cardiol 2008;52:1769-81.

36. Mora S, Glynn RJ, Hsia J, et al. Statins for the primary prevention of cardiovascular events in women with elevated high-sensitivity C-reactive protein or dyslipidemia: results from the Justification for the Use of Statins in Prevention: An Intervention Trial Evaluating Rosuvastatin (JUPITER) and meta-analysis of women from primary prevention trials. Circulation 2010;121:1069-77.

37. Robinson JG. Starting primary prevention earlier with statins. Am J Cardiol 2014;114:1437-42.

38. Hair JF, Black WC, Babin BJ, et al. Multivariate data analysis. 6th edn. Uppersaddle River: Pearson Prentice Hall, 2006.

39. Figueiredo Filho DB, Paranhos R, ECd R, et al. When is statistical significance not significant? Brazilian Political Science Review 2013;7:31-55.

40. Murad MH, Montori VM, loannidis JP, et al. How to read a systematic review and meta-analysis and apply the results to patient care: users guides to the medical literature. JAMA 2014;312:171-9.

41. Yusuf S, Bosch J, Dagenais G, et al. Cholesterol lowering in intermediate-risk persons without cardiovascular disease. $N$ Engl J Med 2016;374:2021-31.

42. Page M, McKenzie J, Dwan K, et al. Bias due to selective inclusion and reporting of outcomes and analyses in systematic reviews of randomised trials of healthcare interventions (Protocol). Cochrane Database Syst Rev 2012:5.
43. Kirkham JJ, Altman DG, Williamson PR. Bias due to changes in specified outcomes during the systematic review process. PLoS One 2010;5:e9810.

44. Krumholz HM. Why data sharing should be the expected norm. BMJ 2015;350:h599.

45. Lo B. Sharing clinical trial data: maximizing benefits, minimizing risk. JAMA 2015;313:793-4.

46. Krumholz HM. Statins evidence: when answers also raise questions. BMJ 2016;354:354.

47. Godlee F. Statins: we need an independent review. BMJ 2016;354:i4992.

48. Collins C. In: Godlee F, ed. CTSU: University of Oxford, 2014.

49. Parish E, Bloom T, Godlee F. Statins for people at low risk. 2015.

50. Byrne P, Cullinan J, Murphy C, et al. Cross-sectional analysis of the prevalence and predictors of statin utilisation in Ireland with a focus on primary prevention of cardiovascular disease. BMJ Open 2018;8:e018524.

51. Wallach-Kildemoes $\mathrm{H}$, Stovring $\mathrm{H}$, Holme Hansen E, et al. Statin prescribing according to gender, age and indication: what about the benefit-risk balance? J Eval Clin Pract 2016;22:235-46.

52. Wallach Kildemoes $H$, Vass M, Hendriksen $C$, et al. Statin utilization according to indication and age: a Danish cohort study on changing prescribing and purchasing behaviour. Health Policy 2012;108:216-27.

53. Feely J, Bennett K. Epidemiology and economics of statin use. Ir Med J 2008;101:188-91.

54. Wallach Kildemoes H, Hendriksen C, Andersen M. Drug utilization according to reason for prescribing: a pharmacoepidemiologic method based on an indication hierarchy. Pharmacoepidemiol Drug Saf 2012;21:1027-35.

55. Godlee F. Statins and The BMJ. BMJ 2014;349:g5038.

56. Getz L, Kirkengen AL, Hetlevik I, et al. Ethical dilemmas arising from implementation of the European guidelines on cardiovascular disease prevention in clinical practice. A descriptive epidemiological study. Scand J Prim Health Care 2004;22:202-8.

57. Cordoba G, Schwartz L, Woloshin S, et al. Definition, reporting, and interpretation of composite outcomes in clinical trials: systematic review. BMJ 2010;341:c3920.

58. Ferreira-González I, Busse JW, Heels-Ansdell D, et al. Problems with use of composite end points in cardiovascular trials: systematic review of randomised controlled trials. BMJ 2007;334:786.

59. Sun X, loannidis JP, Agoritsas T, et al. How to use a subgroup analysis: users' guide to the medical literature. JAMA 2014;311:405-11.

60. Collins R, Reith C, Emberson J, et al. Interpretation of the evidence for the efficacy and safety of statin therapy. Lancet 2016;388.

61. Armitage J. The safety of statins in clinical practice. The Lancet 2007;370:1781-90.

62. Buettner C, Davis RB, Leveille SG, et al. Prevalence of musculoskeletal pain and statin use. J Gen Intern Med 2008;23:1182-6.

63. Fernandez G, Spatz ES, Jablecki C, et al. Statin myopathy: a common dilemma not reflected in clinical trials. Cleve Clin J Med 2011;78:393.

64. Strom BL. Pharmacoepidemiology: John Wiley \& Sons, 2006.

65. Jepsen P, Johnsen SP, Gillman MW, et al. Interpretation of observational studies. Heart 2004;90:956-60.

66. Casula M, Mozzanica F, Scotti L, et al. Statin use and risk of newonset diabetes: a meta-analysis of observational studies. Nutr Metab Cardiovasc Dis 2017;27:396-406.

67. Sattar N, Preiss D, Murray HM, et al. Statins and risk of incident diabetes: a collaborative meta-analysis of randomised statin trials. Lancet 2010;375:735-42.

68. Waters DD, Ho JE, DeMicco DA, et al. Predictors of new-onset diabetes in patients treated with atorvastatin: results from 3 large randomized clinical trials. J Am Coll Cardiol 2011;57:1535-45.

69. Albarqouni L, Doust J, Glasziou P. Patient preferences for cardiovascular preventive medication: a systematic review. Heart. 2017.

70. Byrne P, Cullinan J, Gillespie P. Statins for primary prevention of cardiovascular disease: modelling guidelines and patient preferences based on an Irish cohort. Br J Gen Prac 2019. Forthcoming.

71. Karmali KN, Lloyd-Jones DM, Berendsen MA, et al. Drugs for primary prevention of atherosclerotic cardiovascular disease: an overview of systematic reviews. JAMA Cardiol 2016;1:341-9. 